\title{
Modulated wideband converter with run length limited sequences
}

\author{
Enpin Yanga), Xiao Yan, and Kaiyu Qin \\ School of aeronautics and astronautics, \\ University of Electronic Science and Technology of China, \\ No.2006 Xiyuan Ave, West Hi-Tech Zone, Chengdu, 611731, China \\ a)yang_enpin@163.com
}

\begin{abstract}
The modulated wideband converter (MWC) is a powerful subNyquist sampling system, which can acquire sparse wideband analog signal without prior information of frequency support. In this letter, we aim at enlarging the input bandwidth of MWC under a certain chip rate constraint, which is realized by utilizing run length limited (RLL) sequence in the modulation stage. Simulation results show that when applying our method, input bandwidth of MWC can be improved by $25 \%$ without reconstruction performance loss.
\end{abstract}

Keywords: MWC, sub-Nyquist sampling, RLL sequence, chip rate Classification: Circuits and modules for electronic instrumentation

\section{References}

[1] M. Mishali and Y. C. Eldar: "From theory to practice: Sub-Nyquist sampling of sparse wideband analog signals," IEEE J. Sel. Topics Signal Process. 4 (2010) 375 (DOI: 10.1109/JSTSP.2010.2042414).

[2] M. Mishali and Y. C. Eldar: "Xampling: Signal acquisition and processing in union of subspaces," IEEE Trans. Signal Process. 59 (2011) 4719 (DOI: 10.1109/TSP.2011.2161472).

[3] T. Zahavy, et al:: "Sub-Nyquist sampling of OFDM signals for cognitive radios," IEEE ICASSP (2014) 8092 (DOI: 10.1109/ICASSP.2014.6855177).

[4] M. Mishali and Y. C. Eldar: "Expected-RIP: Conditioning of the modulated wideband converter," IEEE ITW (2009) 343 (DOI: 10.1109/ITW.2009. 5351492).

[5] X. Yang, et al.: "Subsampled circulant matrix based analogue compressed sensing," Electron. Lett. 48 (2012) 767 (DOI: 10.1049/el.2012.0366).

[6] C. H. Lin, et al:: "A novel Sub-Nyquist sampling of sparse wideband signals," IEEE ICASSP (2013) 4628 (DOI: 10.1109/ICASSP.2013.6638537).

[7] L. Chen, et al:: "A calibration system and perturbation analysis for the modulated wideband converter," IEEE ICOSP (2010) 78 (DOI: 10.1109/ ICOSP.2010.5656659).

[8] Y. L. Chen, et al.: "Modulated wideband converter with non-ideal lowpass filters,” IEEE ICASSP (2010) 3630 (DOI: 10.1109/ICASSP.2010.5495912).

[9] T. D. Wang, et al.: "DOA estimation of coherently distributed sources based on block-sparse constraint with measurement matrix uncertainty," IEICE Electron. Express 10 (2013) 20120863 (DOI: 10.1587/elex.10.20120863).

[10] T. Wimalajeewa, et al.: "OMP based joint sparsity pattern recovery under 
communication constraints," IEEE Trans. Signal Process. 62 (2014) 5059 (DOI: 10.1109/TSP.2014.2343947).

[11] E. Yang, et al.: "A greedy pursuit algorithm for arbitrary block sparse signal recovery," IEEE ISCAS (2016) 1858.

[12] A. Harms, et al.: "A constrained random demodulator for Sub-Nyquist sampling," IEEE Trans. Signal Process. 61 (2013) 707 (DOI: 10.1109/TSP. 2012.2231077).

[13] G. Bilardi and R. Padovani: "Spectral analysis of functions of Markov chains with applications," IEEE Trans. Commun. 31 (1983) 853 (DOI: 10.1109/ TCOM.1983.1095910).

\section{Introduction}

The well-known Shannon-Nyquist sampling theorem states that any analog signal bandlimited to $B \mathrm{~Hz}$ can be perfectly reconstructed from its uniform samples if the sampling rate is no less than $2 B \mathrm{~Hz}$ namely the Nyquist rate. However, if the $B$ is extremely big, this sampling method can be hardly achieved, because the required Nyquist rate may exceed the specifications of the best analog-to-digital converter nowadays by orders of magnitude. The modulated wideband converter (MWC) $[1,2,3]$ can solve this problem, when the signal to be sampled is a sparse wideband signal. The most creative feature of MWC is that it can acquire sparse wideband analog signal at a rate far below the Nyquist rate without the prior information of frequency support, while this information must be known in advance for many other sub-Nyquist sampling methods.

The original MWC consists of four parts: modulation stage (including mixers and modulation sequence generators), ideal analog lowpass filters, analog to digital converters and reconstruction algorithm. However, the prototype of MWC is too ideal, thus many researches have modified it to improve its performance or applicability. In [4], different kinds of modulation sequences are tested and constraint conditions of sequences are derived. In [5], the Zadoff-Chu sequences are employed to reduce the complexity of hardware. In [6], a simplified MWC is proposed which has only one channel, but it requires a very complicated filter bank. In [7] and [8], the impact of model mismatch and using non-ideal lowpass filter in MWC are studied respectively, and corresponding compensation algorithms are proposed. Inspired by the block sparsity [9, 10], in our previous work [11], we have developed an algorithm which can be applied for MWC to reconstruct signal without the prior knowledge of the band number.

In this letter, we aim at modifying the input bandwidth problem of MWC, because the input bandwidth of a sampling system is an important parameter in many applications such as passive radar, ultra-wideband communication and cognitive radio, but also because bandwidth of original MWC is constrained by the chip rate of pseudo-random sequence (input bandwidth equals the chip rate and the sequence is hard to generate when required chip rate is excessively high). A pseudo-random sequence consists of two symbols: +1 and -1 , and the chip rate indicates the rate of change of symbols. Since polar transition $(+1$ to -1 or -1 to +1 ) of this kind of sequence is random, chip rate equals the total number of 
symbols (i.e. transition times) in a period time divided by this period of time, for example, in one second, there are $M$ symbols, then chip rate is $M / 1 \mathrm{~Hz}$. Specifically, in our research, the bandwidth can be enlarged without increasing the chip rate by utilizing the run-length-limited (RLL) sequence as the modulation sequence, because RLL sequence constrains the transition of polarity of symbols. We first study how RLL sequences affect the MWC by analyzing their parameters and power spectra. Then, we solve the recovery problem caused by incorporating RLL sequences. Finally, we examine whether RLL sequences has the expected restricted isometry property (ExRIP) which guarantees that sampled signals can be reconstructed with high probability.

\section{Modulated wideband converter}

The MWC is able to process multiband signal whose support resides within $N$ disjoint frequency intervals (the biggest frequency interval cannot exceed $b \mathrm{~Hz}$ and $\mathrm{Nb} \mathrm{Hz}$ is less than the Nyquist rate). And it consists of $m$ channels, as depicted in Fig. 1 [1]. In the $i$-th channel, sampling rate is $1 / T_{S}\left(T_{s}\right.$ is sampling period) and cutoff frequency of filter equals $1 / 2 T_{s}$; the input signal $x(t)$ which is bandlimited to $B \mathrm{~Hz}$ is modulated by a pseudo-random sequence $p_{i}(t)$ with period $T_{p}$. Note that $1 / T_{s}=1 / T_{p}=b \mathrm{~Hz}$ and assume that in one period $T_{p}$ there are $M$ symbols, then $M / T_{p}=2 B \mathrm{~Hz}$, namely chip rate equals the Nyquist rate.

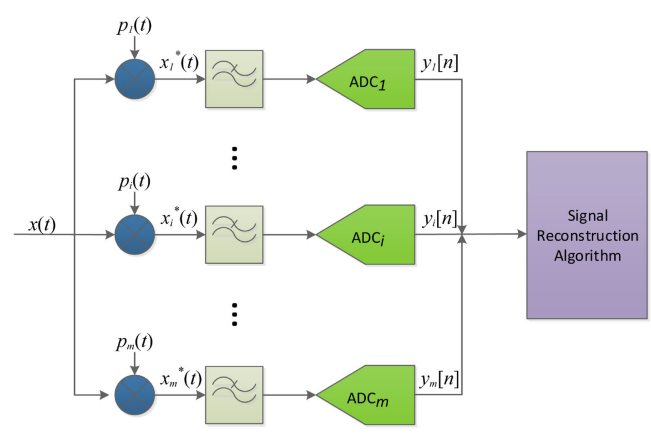

Fig. 1. Block diagram of the MWC. Each $p_{i}(t)$ is a periodical sequence and its waveform alternates at the Nyquist rate.

(a)

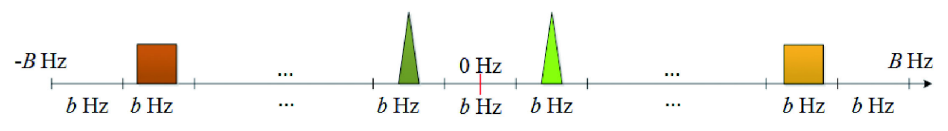

(b)
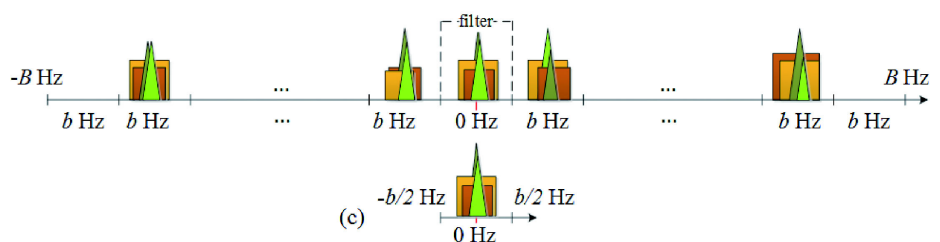

(d)

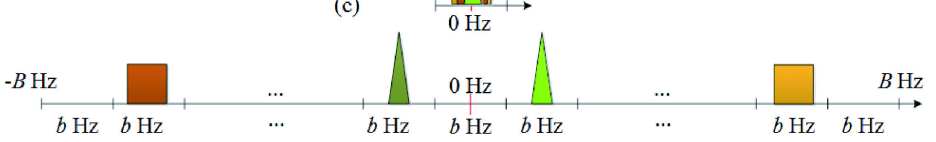

Fig. 2. How the MWC works. (a) Spectrum of the input multiband signal. (b) Spectrum of modulated signal of the $i$-th channel. (c) Filtered signal of the $i$-th channel. (d) The reconstructed signal. 
Fig. 2 shows how the MWC works (note that triangles and quadrangles represent arbitrary spectral slices). Fig. 2(a) is an input signal bandlimited to $B \mathrm{~Hz}$. Fig. 2(b) shows the spectrum of modulated signal of the $i$-th channel. The function of modulation stage is to scramble the original spectrum such that each spectral slice with bandwidth $b \mathrm{~Hz}$ can be transferred to baseband (i.e. $-b / 2 \mathrm{~Hz}$ to $b / 2 \mathrm{~Hz}$ ). Fig. 2(c) indicates the spectrum of filtered signal of the $i$-th channel, and it is a shifted weighted sum of spectrum of input signal. Fig. 2(d) is the reconstructed signal which is calculated based on all filtered signals from all channels.

\section{Analysis of using RLL sequence}

Before we introduce the RLL sequence, two things have to be stated. The first one is why input bandwidth of MWC is decided by the chip rate of pseudo-random sequence. Bandwidth of pseudo-random sequence is equal to its chip rate, and it decides the frequency range in which spectral slices can be transferred into baseband. This frequency range is therefore the input bandwidth of MWC. The other one is how to guarantee the MWC can recover original signal from those shifted weighted sums. One necessary condition is that amplitudes of spectrum of the sequence should be big enough in the entire input bandwidth. The amplitude of a sub-band (e.g. a $b \mathrm{~Hz}$ interval) is the weighted coefficient which determines how much energy of the spectral slice in this sub-band can be transferred into the baseband. If amplitude of one sub-band is small, namely the weighted coefficient is small, then after transferring the corresponding spectral slice could be submerged in noise, and the recovery algorithm can barely reconstruct the signal (signal recovery algorithm is beyond the current scope of this letter, which have been deeply discussed in many compressive sensing literatures; this letter only focuses on utilizing RLL sequences). Therefore, as long as we find a kind of sequence whose spectrum is wider than that of the pseudo-random sequence under a certain chip rate constraint and whose amplitudes are big enough, the input bandwidth can be enlarged.

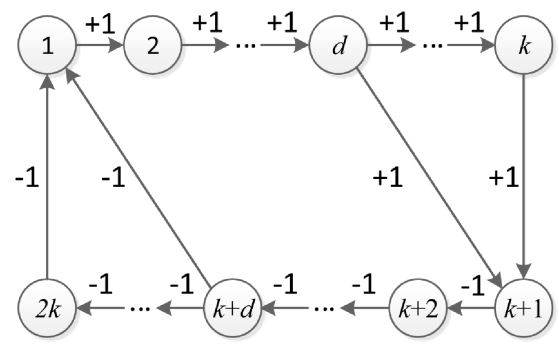

(a) Markov chain of RLL sequences

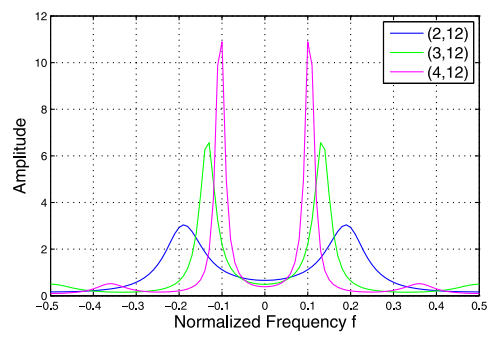

(b) Power spectral of MRS

Fig. 3. Transition diagram and power spectra of MRS.

A $(d, k)$ constrained RLL sequence is a kind of binary sequence, which consists of +1 and -1 . Parameters $(d, k)$ are positive integer, they indicate minimum run length and maximum run length respectively (run length means the symbol number between polar transitions). Minimum run length restricts the chip rate. For example, in a period of time $T$, there are $M$ symbols, chip rate of a pseudo-random sequence is $M / T \mathrm{~Hz}$ and that of a $(d, k) \mathrm{RLL}$ sequence is $M /(d T) \mathrm{Hz}$. The interesting thing is 
that chip rate of pseudo-random sequence is $d$ times greater than that of RLL sequence, but their power spectra have same bandwidth. In other words, for a certain chip rate, using RLL sequence can enlarge input bandwidth. Note that if $d=1$, then $M / T=M /(d T)$, hence the reasonable minimum value of $d$ is 2 .

In the following, we analyze the power spectrum of the MRS to evaluate whether it is suitable for MWC. The RLL sequence can be generated by a markov chain. Fig. 3 shows the transition diagram of markov-generated RLL sequences (MRS) [12] and a set of power spectra [13] of MRSs with different $d$, which are normalized according to their bandwidth. For each MRS in Fig. 3(b), three coordinates of the frequency axis should be studied. The first coordinate is $f_{1}=0.5 / d$ which denotes the normalized chip rate of $(d, k)$ MRS; the second is $f_{2 \_}=0.5$ which is the bandwidth of MRS; the last one is $f_{2 \_}$whose value is also 0.5 and it indicates the theoretical maximal input bandwidth if this MRS is applied to MWC. As shown in Fig. 3(b), with the increasing of $d$, energy of MRS becomes more and more centralized; when $d=4$, it is concentrated around $f=0.1$, and almost all amplitudes of high frequency sub-band $(f>0.2)$ are negligible. As a result, spectral slices, which reside within the high frequency sub-band of input signal, cannot be reconstructed. The relatively flat spectrum is obtained when $d=2$. Therefore, $(2, k)$ MRS is the best choice to implement our modification, though it still has the negligible amplitude problem in the interval $(0.3,0.5)$.

Intuitively, if all weighted coefficients (i.e. amplitudes of the power spectrum) are big enough, the reconstruction issue can be solved. This idea can be realized by cutting off the power spectrum of $(2, k)$ MRS. If $f_{2 \_2}$ can slide toward $f_{1}\left(f_{2 \_}\right.$is fixed), the spectrum of MRS can be treated as being cutoff so that negligible values in the high frequency sub-band are excluded. The price for cutoff is that the improvement of input bandwidth cannot achieve the theoretical bound: twice the original one. Note that $f_{2 \_}$cannot slide over the point $f_{1}=0.25(d=2)$, otherwise input bandwidth of MWC cannot be enlarged, therefore, $f_{2 \_}$resides in interval $(0.25,0.5)$. For the sake of brevity, we use $f$ to denote $f_{2 \_}$hereafter. The relationship among chip rate of MRS $f_{R L L}$, input bandwidth of MWC $f_{N Y Q}$ and $f$ is

$$
f_{N Y Q}=4 f f_{R L L}
$$

Eq. (1) is derived from $f_{R L L}=0.5 / d,\left(f_{N Y Q} / 2\right) / f=0.5$ and $d=2$.

Obviously, we must work out the value of $f$, in other word, we need to define the negligible amplitude of power spectrum. However, it is hard to estimate what amplitude in frequency interval $(0.25,0.5)$ is negligible under different SNR conditions. Considering that the recovery performance of original MWC gets worse when SNR $<-5 \mathrm{~dB}$, we can choose a threshold to resolve this issue under the worst situation $\mathrm{SNR}=-5 \mathrm{~dB}$.

As shown in Fig. 4, in sub-band [0, 0.25], the minimal amplitude of power spectral of MRSs emerges at the origin $P_{(2, k)}(0)$, where $P_{(2, k)}(z)$ denotes amplitude of the power spectrum of $(2, k)$ MRS when frequency is $z$; in sub-band $(0.25,0.5]$, amplitudes decrease progressively. We have tested $P_{(2, k)}(0)$ by extensive simulation, it is big enough to recover the input signal, when $\mathrm{SNR}=-5 \mathrm{~dB}$. Naturally, when a $(2, k)$ MRS is applied, the corresponding $P_{(2, k)}(0)$ can be chosen as a threshold (it may be not the optimal one) to distinguish negligible amplitudes. 


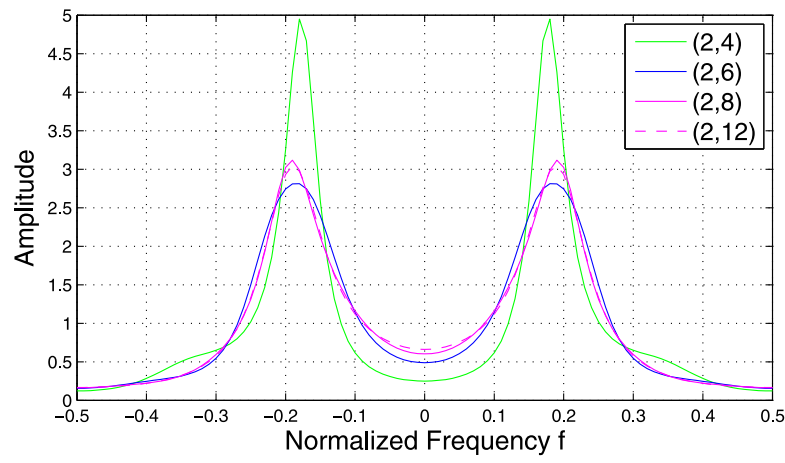

Fig. 4. Power spectra of $(2, k)$ MRSs

Consequently, $f$ can be easily found out,

$$
P_{(2, k)}(f)=P_{(2, k)}(0) \quad f \in(0.25,0.5)
$$

Once the $f$ is figured out, the input bandwidth improvement ratio compared with original MWC can be derived

$$
\text { ratio }=\left(f-f_{1}\right) / f_{1}
$$

Table I. Input bandwidth improvement ratio when using $P_{(2, k)}(f)$ as threshold.

\begin{tabular}{|l|c|c|}
\hline$(2, \boldsymbol{k})$ & $\boldsymbol{f}$ of $P_{(2, k)}(f)$ & ratio (\%) \\
\hline$(2,6)$ & 0.3075 & 23.00 \\
\hline$(2,8)$ & 0.3000 & 20.00 \\
\hline$(2,10)$ & 0.2924 & 16.96 \\
\hline$(2,12)$ & 0.2912 & 16.48 \\
\hline$(2,15)$ & 0.2910 & 16.40 \\
\hline$(2,20)$ & 0.2900 & 16.00 \\
\hline
\end{tabular}

Table I shows when using corresponding $P_{(2, k)}(0)$ as the threshold the improvement ratio is small. For that reason, a relatively smaller threshold is preferred. As shown in Fig. 4, $P_{(2, k)}(0)$ is a monotone increasing function of $k$. By testing different $P_{(2, k)}(0)$, we discover that the amplitude of $P_{(2,6)}(0)$ is adequate as the threshold when SNR $=-5 \mathrm{~dB}$. Thus Eq. (2) can be revised as

$$
P_{(2, k)}(f)=P_{(2,6)}(0) \quad f \in(0.25,0.5)
$$

Table II. Input bandwidth improvement ratio when $P_{(2,6)}(f)$ as threshold.

\begin{tabular}{|l|c|c|}
\hline $\mathbf{( 2 , \boldsymbol { k } )}$ & $\boldsymbol{f}$ of $P_{(2,6)}(f)$ & ratio (\%) \\
\hline$(2,6)$ & 0.3075 & 23.00 \\
\hline$(2,8)$ & 0.3145 & 25.80 \\
\hline$(2,10)$ & 0.3127 & 25.08 \\
\hline$(2,12)$ & 0.3126 & 25.04 \\
\hline$(2,15)$ & 0.3126 & 25.04 \\
\hline$(2,20)$ & 0.3126 & 25.04 \\
\hline
\end{tabular}


Table II, Eq. (1) and Eq. (3) show that $(2, k)$ MRSs can enlarge the input bandwidth. For the original MWC $f_{N Y Q}$ equals chip rate of pseudo-random sequence, after our modification $f_{N Y Q}=4 f f_{R L L}$, as long as $f>0.25$, the input bandwidth is bigger than the original one. For example, when chip rate is $10 \mathrm{GHz}$, input bandwidth of original MWC is $10 \mathrm{GHz}$, while the bandwidth is about $12.5 \mathrm{G}$ when $(2, k)$ MRS is used. Note that when SNR $>-5 \mathrm{~dB}$, the input bandwidth can be further improved by choosing other thresholds, but in this letter, we only present the worst situation of SNR.

Now we illustrate how to calculate the symbol number $M$ of MRS in a single period $T_{r l l}$ which is a very important parameter of MWC. The value of $M$ is associated to chip rate. According to the second paragraph of this section and Eq. (1), the chip rate of $(2, k)$ MRS can be formulated as

$$
f_{R L L}=\frac{1}{2} \times \frac{f_{N Y Q}}{2 f}=\frac{1}{2} \times \frac{M}{T_{r l l}}
$$

We can derive that

$$
M \geq M_{\min }=\left\lceil\frac{f_{N Y Q} T_{r l l}}{2 f}\right\rceil \text { or } \quad M \geq M_{\min }=\left\lceil 2 T_{\text {rll }} f_{R L L}\right\rceil
$$

Where $\lceil a\rceil$ is a ceiling operator, it returns the greater or equal integer which is closest to $a, M_{\min }$ is the smallest integer that can be chosen as the $M$. Both of the two formula can be used to calculate the $M$, it depends on which parameters are known in advance.

\section{Simulation}

First of all we verify whether the $(2, k)$ MRS has the ExRIP [4] which guarantees the sampled signal can be reconstructed. ExRIP is related to three coefficients, as long as these coefficients are small enough, the MRS satisfies the ExRIP with high probability.

$$
\begin{aligned}
& \alpha(\mathbf{S})=\frac{1}{(m M)^{2}} \sum_{i, k=1}^{m}\left(\mathbf{S}_{i}^{T} \mathbf{S}_{k}\right)^{2} \\
& \beta(\mathbf{S})=\frac{1}{m^{2} M^{3}} \sum_{i, k=1}^{m}\left\|\mathbf{S}_{i} \odot \mathbf{S}_{k}\right\|^{2} \\
& \gamma(\mathbf{S})=\frac{1}{(m M)^{2}} \sum_{i, k=1}^{m}\left(\mathbf{S}_{i}^{T} \mathbf{S}_{k}^{-}\right)^{2}
\end{aligned}
$$

where $m$ is the channel number, $M$ is the symbol number in one period, $\mathbf{S}_{i}$ is the $i$-th MRS, $\odot$ denotes cyclic convolution and $\mathbf{S}_{i}{ }_{i}[n]=\mathbf{S}_{i}[-n]$.

When $(2, k)$ MRS sequence is employed, values of Eq. (7) with different $k$ are calculated (depicted in Fig. 5); provided that chip rate is $10 \mathrm{GHz}$, under original MWC setting $M=195$, after modification, $M=387$. Apparently, in each figure, the value of blue line is smaller than that of the other one, in other words, our modification provides smaller $\alpha(\mathbf{S}), \beta(\mathbf{S})$ and $\gamma(\mathbf{S})$, and it can be seen that all curves become relatively stable when $k \geq 12$, therefore any $(2, k \geq 12)$ MRSs can be applied to MWC. 


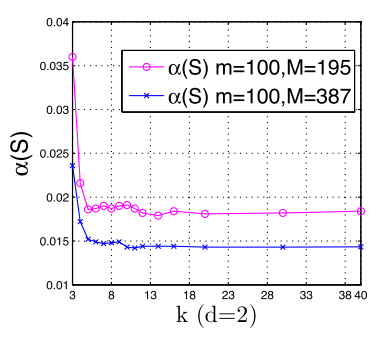

(a) $\alpha(\mathbf{S})$

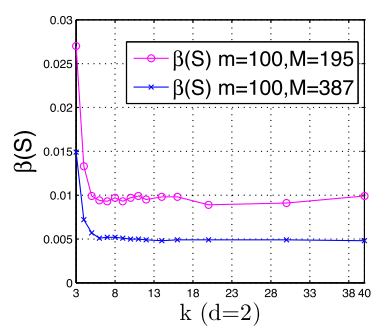

(b) $\beta(\mathbf{S})$

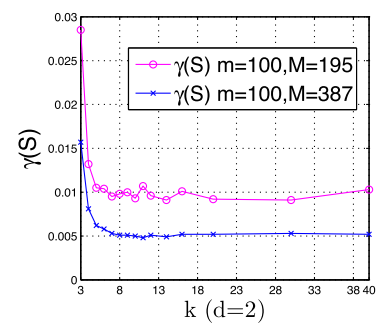

(c) $\gamma(\mathbf{S})$

Fig. 5. ExRIP related coefficients.

After that we implement two simulations to prove our modification is valid. Simulation conditions are set as follows:

1. Original MWC uses pseudo-random sequences and the revised MWC uses $(2,12)$ MRS in the modulation stage;

2. Chip rates of these two kinds of sequences are $10 \mathrm{GHz}$;

3. Spectral slices of each input signal occupies 6 sub-bands (locations of subbands are randomly generated and unknown in advance);

4. Maximum bandwidth of each sub-band (i.e. the $b \mathrm{~Hz}$ interval) of the input multiband signal is $51.3 \mathrm{MHz}$;

5. Both of the sampling rate $1 / T_{s}$ of each channel and the modulation sequence frequency (i.e. $1 / T_{p}$ and $1 / T_{r l l}$ ) are $51.3 \mathrm{MHz}$;

6. The signal reconstruction algorithm is orthogonal matching pursuit.

In the first simulation, we demonstrate that reconstruction performance of the revised MWC is as good as the original one. In this case, bandwidth of input signal is set as $10 \mathrm{GHz}$ which is determined by the original MWC. In Fig. 6, the horizontal axis is the channel number $m$ from 20 to 80 and the vertical axis is the recovery probability which is calculated from successful recovery times divided by total simulation times. In Fig. 6, each point is based on 500 times simulation. We can see that when $\mathrm{SNR}=10 \mathrm{~dB}$ or $0 \mathrm{~dB}$, their recovery probability curves are almost overlapped; in the worst condition where $\mathrm{SNR}=-5 \mathrm{~dB}$, the recovery probability of both the original and revised MWC can reach the same numerical value, when sampling channel $\mathrm{m}>70$. It shows that the revised MWC also has a good recovery performance.

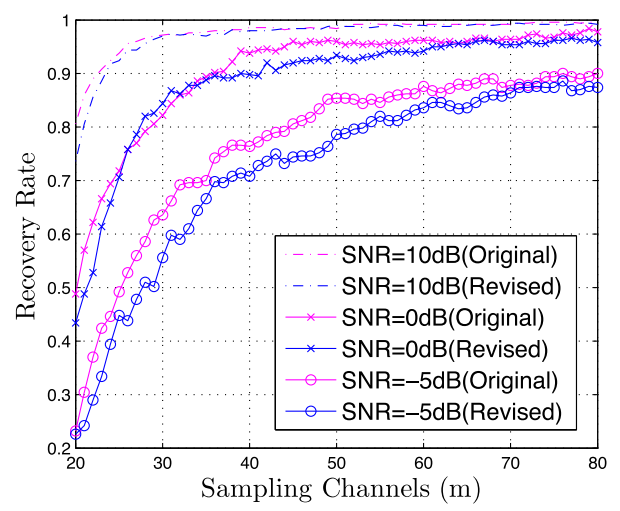

Fig. 6. Comparison of recovery performance 
In the second simulation, we prove that our modification can enlarge the input bandwidth of MWC. As depicted in Fig. 7, revised MWC can still reconstruct input signal with high probability while the original one can hardly reconstruct input signal. In this case, all the simulation settings are similar with the previous one, except that bandwidth of input signal is $12.5 \mathrm{GHz}$ (note that chip rates of both two kind of sequences are still $10 \mathrm{GHz}$ ). Since input signal is randomly generated, it is possible that its frequency support resides within $10 \mathrm{GHz}$. Therefore, this class of signal still can be recovered by the original MWC. Indeed, there must be a lot of randomly generated signals whose support reside in interval [10 GHz, $12.5 \mathrm{GHz}]$, and original MWC cannot recover these signals due to the input bandwidth limitation, that is why the recovery probability curve severely declines compared with the outcome of the previous simulation. It proves that using RLL sequence can enlarge the input bandwidth of MWC under a certain chip rate constraint.

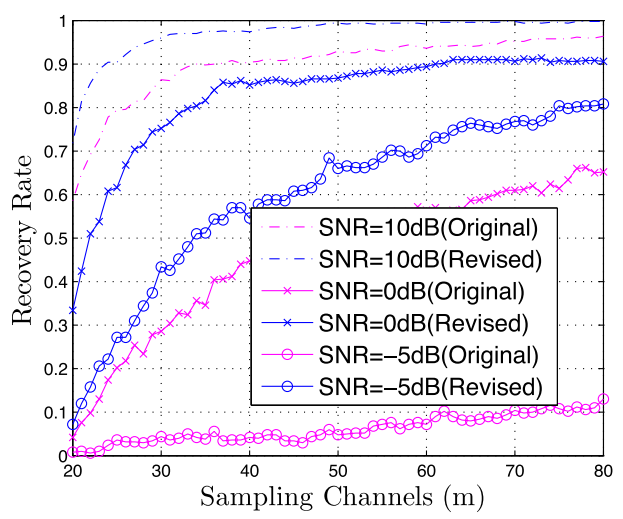

Fig. 7. Comparison of input bandwidth

There is another important issue we should address. We have simulated up to 80 sampling channels for the MWC, however it is virtually impossible to build such a multitude of ADC channels in hardware. Fortunately, there is a solution, which is elaborated in [1], to reduce the required hardware channel by increasing computational complexity.

\section{Conclusion}

In this letter, we present a method to enlarge the input bandwidth of MWC and solve the negligible amplitude problem caused by using RLL sequence. We have analyzed how the RLL sequence affects the MWC. And we have shown how to choose appropriate parameters of the $(d, k)$ RLL sequence by studying its power spectrum and the ExRIP. Simulation results show that our modification can enlarge about $25 \%$ of the input bandwidth without reconstruction performance loss. 